\title{
Non-concave utility maximisation on the positive real axis in discrete time
}

ARTICLE in MATHEMATICS AND FINANCIAL ECONOMICS · JANUARY 2015

DOI: 10.1007/s11579-015-0146-4 · Source: arXiv

READS

8

3 AUTHORS, INCLUDING:

Laurence Carassus

Université de Reims Champagne-Ardenne

20 PUBLICATIONS 73 CITATIONS

SEE PROFILE 


\title{
Non-concave utility maximisation on the positive real axis in discrete time*
}

\author{
Laurence Carassus ${ }^{\dagger} \quad$ Miklós Rásonyi ${ }^{\ddagger} \quad$ Andrea M. Rodrigues ${ }^{\S}$
}

30th September 2014

\begin{abstract}
We treat a discrete-time asset allocation problem in an arbitrage-free, generically incomplete financial market, where the investor has a possibly non-concave utility function and wealth is restricted to remain non-negative. Under easily verifiable conditions, we establish the existence of optimal portfolios.
\end{abstract}

Keywords: Discrete-time models ; Dynamic programming ; Finite horizon ; Incomplete markets ; Non-concave utility ; Optimal portfolio.

AMS MSC 2010: Primary 93E20, 91B70, 91B16, Secondary 91G10.

\section{Introduction}

We consider investors trading in a multi-asset and discrete-time financial market who are aiming to maximise their expected utility from terminal wealth. If the utility function is defined on the non-negative half-line, is concave, and the problem has a finite value function, then there is always such a strategy, see Rásonyi and Stettner [19]. In the present paper, we provide (mild) sufficient conditions on a possibly non-concave utility function which guarantee the existence of an optimal strategy.

This problem has recently been addressed in Reichlin [20] in a continuous-time setting, but assuming a complete market, just like in the studies Berkelaar, Kouwenberg and Post [1]; Carassus and Pham [4]. The incomplete case is largely unexplored territory: in the present setting we are aware of Chapter IV of Reichlin [21] and Rásonyi and Rodrigues [15] only, where existence results were proved for some very specific market models.

By treating multi-step discrete-time markets, the present paper is the first to cover a substantial class of incomplete models which can be fitted to arbitrary econometric data.

The case of utilities defined on the whole real line was treated in Carassus and Rásonyi [5], while probability distortions (in the spirit of cumulative prospect theory) were studied in Carassus and Rásonyi [6]; Rásonyi and Rodríguez-Villarreal [17] in the discrete-time setting.

Continuous-time studies involving non-concave utilities include Campi and Del Vigna [3]; Carlier and Dana [7]; Jin and Zhou [11]; Rásonyi and Rodrigues [16], but these references all make the assumption that the market is complete.

\footnotetext{
${ }^{*}$ L. Carassus thanks LPMA (UMR7599) for support. A. M. Rodrigues gratefully acknowledges the financial support of FCT - Fundação para a Ciência e Tecnologia (Portuguese Foundation for Science and Technology) through the Doctoral Grant SFRH/BD/69360/2010. Part of this research was carried out while M.R. and A.M.R. were affiliated with the School of Mathematics, University of Edinburgh.

${ }^{\dagger}$ LMR, Université de Reims Champagne-Ardenne, Reims, France. E-mail: laurence.carassus@univ-reims.fr

¥Alfréd Rényi Institute of Mathematics, Hungarian Academy of Sciences, Budapest, Hungary. E-mail: rasonyi@renyi.mta.hu

$\S$ Dublin City University, Dublin, Ireland. E-mail: Andrea.MeirelesRodrigues@dcu.ie
} 
A brief outline of this manuscript is as follows. Section 2 is dedicated to specifying the market model and to introducing the relevant notation. In Section 3 we formulate our main result. Next, in Section 4 we examine the problem in a one-step setting, whilst in Section 5 we prove our main result, using a dynamic programming approach. For the sake of a simple presentation, the proofs of some technical results are collected in Appendix A.

\section{Notation and set-up}

\subsection{The market}

In what follows, we shall consider a frictionless and totally liquid financial market model with finite trading horizon $T \in \mathbb{N}$, in which the current time is denoted by 0 and trading is assumed to occur only at the dates $\{0,1, \ldots, T\}$.

As usual, the uncertainty in the economy is characterised by a complete probability space $(\Omega, \mathscr{F}, \mathbb{P})$, where $\mathscr{F}$ is a $\sigma$-algebra on the sample space $\Omega$, and $\mathbb{P}$ is the underlying probability measure (to be interpreted as the physical probability). Moreover, all the information accruing to the agents in the economy is described by a discrete filtration $\mathbb{F}=\left\{\mathscr{F}_{t} ; t \in\{0,1, \ldots, T\}\right\}$ such that $\mathscr{F}_{0}$ contains all $\mathbb{P}$-null sets. Finally, we assume for convenience that the $\sigma$-algebra $\mathscr{F}_{0}$ is $\mathbb{P}$-trivial, and also that $\mathscr{F}=\mathscr{F}_{T}$.

Next, we fix a strictly positive integer $d$ and consider a process $S=\left\{S_{t} ; t \in\{0,1, \ldots, T\}\right\}$, so that $S_{t}$ represents the time- $t$ prices of $d$ traded risky assets. Denoting by $\Xi_{t}^{n}$ the family of all $\mathscr{F}_{t}$-measurable random vectors $\xi: \Omega \rightarrow \mathbb{R}^{n}$ for each $n \in \mathbb{N}$ and each $t \in\{0,1, \ldots, T\}$, we assume that $S_{t} \in \Xi_{t}^{d}$ for every $t \in\{0,1, \ldots, T\}$, i.e., $S$ is $\mathbb{F}$-adapted. We shall also assume, without loss of generality, that the risk-free asset in this economy has constant price equal to one at all times. Finally, for each $t \in\{1, \ldots, T\}$, we define $\Delta S_{t} \triangleq S_{t}-S_{t-1}$.

We recall that a self-financing portfolio is a process $\phi=\left\{\phi_{t} ; t \in\{1, \ldots, T\}\right\}$, with $\phi_{t} \in$ $\Xi_{t-1}^{d}$ for all $t \in\{1, \ldots, T\}$, and its wealth process $\Pi^{\phi}=\left\{\Pi_{t}^{\phi} ; t \in\{0,1, \ldots, T\}\right\}$ satisfies, for every $t \in\{1, \ldots, T\}$,

$$
\Pi_{t}^{\phi}=\Pi_{0}^{\phi}+\sum_{s=1}^{t}\left\langle\phi_{s}, \Delta S_{s}\right\rangle \text { a.s. }
$$

We denote by $\Phi$ the class of all self-financing portfolios. In addition, we shall impose the trading constraint that the value of a portfolio should not be allowed to become strictly negative, so we say that a portfolio $\phi \in \Phi$ is admissible for $x_{0} \geq 0$ (and we write $\phi \in \Psi\left(x_{0}\right)$ ) if, for every $t \in\{1, \ldots, T\}$, the inequality $\Pi_{t}^{\phi} \geq 0$ holds a.s. with $\Pi_{0}^{\phi}=x_{0}$. Such a constraint is natural and frequently imposed, see e.g. [14; 19].

No investor should be allowed to make a profit out of nothing and without risk.

Assumption 2.1. The market does not admit arbitrage, i.e.,

$$
\text { for all } x_{0} \geq 0 \text {, if } \phi \in \Psi\left(x_{0}\right) \text { with } \Pi_{T}^{\phi} \geq x_{0} \text { a.s., then } \Pi_{T}^{\phi}=x_{0} \text { a.s.. }
$$

Now fix $t \in\{1, \ldots, T\}$. We know that there exists a regular conditional distribution of $\Delta S_{t}$ with respect to $\mathscr{F}_{t-1}$ under the physical measure $\mathbb{P}$, which we shall denote by $\mathbb{P}^{\Delta S_{t} \mid \mathscr{F}_{t-1}}$.

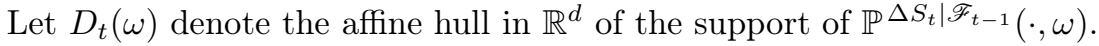

Under the no-arbitrage Assumption 2.1, it follows from Theorem 3 in Jacod and Shiryaev [10] that $D_{t}(\omega)$ is actually a linear space for $\mathbb{P}$-almost every $\omega$.

Furthermore, for each fixed $t \in\{1, \ldots, T\}$, we define two important families of functions. Firstly, given any $\mathscr{F}_{t-1}$-measurable random variable $H \geq 0$ a.s., we set

$$
\Xi_{t-1}^{d}(H) \triangleq\left\{\xi \in \Xi_{t-1}^{d}: H+\left\langle\xi, \Delta S_{t}\right\rangle \geq 0 \text { a.s. }\right\} \text {. }
$$

In the particular case where $H=x$ a.s. for some $x \geq 0$, we have

$$
\Xi_{t-1}^{d}(x) \triangleq\left\{\xi \in \Xi_{t-1}^{d}: x+\left\langle\xi, \Delta S_{t}\right\rangle \geq 0 \text { a.s. }\right\} .
$$


On the other hand, we take $\widetilde{\Xi}_{t-1}^{d}$ to be the class of all random vectors $\xi \in \Xi_{t-1}^{d}$ such that $\xi(\omega) \in D_{t}(\omega)$ for $\mathbb{P}$-a.e. $\omega$. The notations $\tilde{\Xi}_{t-1}^{d}(x), \tilde{\Xi}_{t-1}^{d}(H)$ are self-explanatory.

Proposition 2.2. The following two statements are equivalent,

(i) (NA) holds true.

(ii) For every $t \in\{1, \ldots, T\}$, there exist $\mathscr{F}_{t-1}$-measurable random variables $\beta_{t}>0, \kappa_{t}>0$ a.s. such that, for every $\xi \in \widetilde{\Xi}_{t-1}^{d}$, the inequality

$$
\mathbb{P}\left(\left\langle\xi, \Delta S_{t}\right\rangle \leq-\beta_{t}\|\xi\| \mid \mathscr{F}_{t-1}\right) \geq \kappa_{t}
$$

holds a.s. on $\left\{D_{t}(\omega) \neq\{0\}\right\}$.

Proof. This is Proposition 3.3 in Rásonyi and Stettner [18].

Remark 2.3. We notice that the above 'quantitative' characterisation of (NA) holds true only for $\mathscr{F}_{t-1}$-measurable, $\mathbb{R}^{d}$-valued functions $\xi$ which belong to $D_{t}$ a.s.. This will motivate the use of orthogonal projections later on (cf. Section 4).

\subsection{The investor}

Investors' risk preferences are described by a (possibly non-concave) utility function.

Definition 2.4 (Non-concave random utility). A random utility (on the non-negative half-line) is any function $u:(0,+\infty) \times \Omega \rightarrow \mathbb{R}$ verifying the following two properties,

(i) for every $x \in(0,+\infty)$, the function $u(x, \cdot): \Omega \rightarrow \mathbb{R}$ is $\mathscr{F}$-measurable,

(ii) for every $\omega \in \Omega$, the function $u(\cdot, \omega):(0,+\infty) \rightarrow \mathbb{R}$ is non-decreasing and continuous.

For each $\omega \in \Omega$, we set $u(0, \omega) \triangleq \lim _{x \downarrow 0} u(x, \omega)$. Note that $u(0, \omega)$ may take the value $-\infty$.

Remark 2.5. As in this paper we restrict wealth to be non-negative, we consider utilities which are defined only over the non-negative real line. Continuity and monotonicity are standard assumptions. Also, as $u$ will be used to assess the future wealth of the investor, it may well depend on economic variables and hence it can be random, see Example 2.8 below. Lastly, unlike most studies, we do not assume concavity or smoothness of $u$.

We proceed by noticing that, since for every $\omega \in \Omega, u(\cdot, \omega)$ is a monotone function, the limit $u(+\infty, \omega) \triangleq \lim _{x \rightarrow+\infty} u(x, \omega)$ exists (though it may not be finite). We shall require the following.

Assumption 2.6. The negative part of $u$ at 0 has finite expectation, i.e., ${ }^{1}$

$$
\mathbb{E}_{\mathbb{P}}\left[u^{-}(0, \cdot)\right]<+\infty .
$$

In the sequel we shall often omit the dependence of $u$ on $\omega$ in the notation.

Remark 2.7. If $u$ is deterministic, then the above assumption is equivalent to $u(0)>-\infty$. This is admittedly restrictive, as it excludes that $u(x)$ behaves like $\log (x)$ or $-x^{\alpha}$ (with $\alpha<0$ ) in the vicinity of 0 . It still allows, however, a very large class of utilities.

We continue this subsection with an important example of a random utility function, in the spirit of cumulative prospect theory (CPT, see Kahneman and Tversky [13]; Tversky and Kahneman [22]).

\footnotetext{
${ }^{1}$ Here $x^{+} \triangleq \max \{x, 0\}$ and $x^{-} \triangleq-\min \{x, 0\}$ for every $x \in \mathbb{R}$. Furthermore, in order to make the notation less heavy, given any function $f: X \rightarrow \mathbb{R}$, we shall write henceforth $f^{ \pm}(x) \triangleq[f(x)]^{ \pm}$for all $x \in X$.
} 
Example 2.8 (Reference point). Within the CPT framework, every investor is assumed to have a reference point in wealth (also referred to in the literature as benchmark or status quo, see e.g. Bernard and Ghossoub [2], He and Zhou [9], Carassus and Rásonyi [6]), with respect to which payoffs at the terminal time $T$ are evaluated. Therefore, the investors' decisions are not based on the terminal level of wealth (as it is assumed in the Expected Utility Theory of von Neumann and Morgenstern [23]), but rather on the deviation of that wealth level from the reference point.

Mathematically, a reference point is any fixed scalar-valued and $\mathscr{F}$-measurable random variable $B \geq 0$ a.s. Thus, given a payoff $X$ at the terminal time $T$ and a scenario $\omega \in \Omega$, the investor is said to make a gain (respectively, a loss) if the deviation from the reference level is strictly positive (respectively, strictly negative), that is, $X(\omega)>B(\omega)$ (respectively, $X(\omega)<B(\omega))$.

Note that $B$ may be taken to be, for example, a non-negative constant (this is the case in Berkelaar, Kouwenberg and Post [1]; Bernard and Ghossoub [2]). The reference point can also be stochastic (for instance, to reflect the fact that the investors compare their performance to that of another investor acting in a perhaps different market).

In this setting, the investor has a random utility defined as

$$
u(x, \omega) \triangleq \widetilde{u}(x-B(\omega)), \quad x>0, \omega \in \Omega,
$$

with $\widetilde{u}: \quad(-\operatorname{ess} \sup B,+\infty) \rightarrow \mathbb{R}$ a (deterministic) non-decreasing and continuous function satisfying $\widetilde{u}(-\operatorname{ess} \sup B)>-\infty\left(\right.$ where we $\operatorname{set} \widetilde{u}(-\operatorname{ess} \sup B) \triangleq \lim _{x \downarrow}-\operatorname{ess} \sup B \widetilde{u}(x)$, as before). Obviously, $\mathbb{E}_{\mathbb{P}}\left[u^{-}(0, \cdot)\right]<+\infty$, so Assumption 2.6 is true for $u$.

We shall make the following assumption on the growth of the function $u$.

Assumption 2.9. There exist constants $\bar{\gamma}>0$ and $\bar{x}>0$, as well as a random variable $c \geq 0$ with $\mathbb{E}_{\mathbb{P}}[c]<+\infty$, such that

$$
u(\lambda x) \leq \lambda^{\bar{\gamma}} u(x)+\lambda^{\bar{\gamma}} c,
$$

for all $\omega \in \Omega, \lambda \geq 1, x \geq \bar{x}$ and $\mathbb{E}_{\mathbb{P}}\left[u^{+}(\bar{x})\right]<\infty$.

Remark 2.10. For $u$ deterministic, concave and continuously differentiable, we recall that

$$
A E_{+}(u) \triangleq \limsup _{x \rightarrow+\infty} \frac{x u^{\prime}(x)}{u(x)}
$$

denotes the asymptotic elasticity of $u$ at $+\infty$ (see Kramkov and Schachermayer [14, p. 943]), and that in this case we always have $A E_{+}(u) \leq 1$ (the reader is referred to Kramkov and Schachermayer [14, Lemma 6.1]).

If, in addition, $u(+\infty) \geq 0$, then we know by Lemma 6.3 in Kramkov and Schachermayer [14] that $A E_{+}(u)$ equals the infimum of all real numbers $\gamma>0$ for which there exists some $\bar{x}>0$ such that, for all $\lambda \geq 1$ and all $x \geq \bar{x}$,

$$
u(\lambda x) \leq \lambda^{\gamma} u(x) .
$$

This latter definition makes sense for possibly non-concave $u$ and arbitrary $\gamma>0$ as well, so following Carassus and Rásonyi [5] we may define the asymptotic elasticity at $+\infty$ of $u$ as

$A E_{+}(u) \triangleq \inf \left\{\gamma>0: \exists \bar{x} \geq 0\right.$ such that $\left.u(\lambda x, \omega) \leq \lambda^{\gamma} u(x, \omega), \forall \omega \in \Omega, \forall \lambda \geq 1, \forall x \geq \bar{x}\right\}$,

with the usual convention that the infimum of the empty set is $+\infty$.

Hence, using this generalized notion of asymptotic elasticity, we see that condition (2.4) holds under Assumption 2.6 if either $u$ is nonnegative and bounded above (by a random constant $c$ ), or $A E_{+}(u) \leq \bar{\gamma}$.

We note that, if $u$ is deterministic, concave and bounded, then $A E_{+}(u) \leq 0$ (again by Kramkov and Schachermayer [14, Lemma 6.1]), but this fails in the non-concave case (see 
Example A.1 below). This shows that having finite asymptotic elasticity, despite being sufficient, is not a necessary condition for a function to verify Assumption 2.9.

Besides, it follows as in Lemma 6.3 of Kramkov and Schachermayer [14] that, for a continuously differentiable (but not necessarily concave) deterministic $u$ having $A E_{+}(u) \leq \gamma$ for some $\gamma>0$ is still equivalent to $\limsup _{x \rightarrow+\infty} x u^{\prime}(x) / u(x) \leq \gamma$.

From this, we immediately get that $A E_{+}(u)<+\infty$ (and hence Assumption 2.9 holds) if $u$ is deterministic, continuously differentiable and if there exists some $p>0$ such that

$$
0<\liminf _{x \rightarrow+\infty} \frac{u^{\prime}(x)}{x^{p}} \leq \limsup _{x \rightarrow+\infty} \frac{u^{\prime}(x)}{x^{p}}<+\infty
$$

Indeed, if the above condition is true for $u$, then on the one hand it is possible to find $m>0$ for which there exists some $\underline{x}>0$ such that $u^{\prime}(x)>m x^{p}$ for all $x \geq \underline{x}$. But this implies that, for all $x \geq \underline{x}$,

$$
u(x) \geq \int_{\underline{x}}^{x} u^{\prime}(y) d y \geq m \frac{x^{p+1}-\underline{x}^{p+1}}{p+1} .
$$

On the other hand, we can find $M>0$ for which there is $\bar{x}>0$ such that $u^{\prime}(x)<M x^{p}$ for all $x \geq \bar{x}$. Defining $\widehat{x} \triangleq \max \{\underline{x}, \bar{x}\}>0$, and combining the preceding inequalities finally gives

for all $x \geq \widehat{x}$, therefore

$$
\frac{x u^{\prime}(x)}{u(x)} \leq(p+1) \frac{M}{m} \frac{x^{p+1}}{x^{p+1}-\underline{x}^{p+1}}
$$

$$
\limsup _{x \rightarrow+\infty} \frac{x u^{\prime}(x)}{u(x)}<+\infty .
$$

In particular, if $u^{\prime}(x)$ is asymptotically equivalent to a power function (that is, $u^{\prime}(x) / x^{p} \rightarrow 1$, $x \rightarrow \infty$ ) then Assumption 2.9 holds. A multitude of piecewise concave or $S$-shaped functions (not only piecewise power functions) can be accomodated in this way, such as the ones considered in Berkelaar, Kouwenberg and Post [1]; Carassus and Pham [4]; Jin and Zhou [11]; Rásonyi and Rodrigues [15].

At last, suppose that $u$ is the utility of Example 2.8. If the conditions below are satisfied:

(i) $\operatorname{ess} \sup B<+\infty$,

(ii) there exist real numbers $\bar{\gamma}>0, \widetilde{x}>0$ and $C \geq 0$ such that, for all $\lambda \geq 1$ and all $x \geq \widetilde{x}$,

$$
\widetilde{u}(\lambda x) \leq \lambda^{\bar{\gamma}} \widetilde{u}(x)+\lambda^{\bar{\gamma}} C,
$$

(iii) the function $\widetilde{u}$ is continuously differentiable on its domain, and there are real numbers $K>0$ and $\widehat{x}>0$ such that, for all $x \geq \widehat{x}$,

$$
\widetilde{u}^{\prime}(x) \leq K
$$

then $u$ fullfills Assumption 2.9. Indeed, setting $\bar{x} \triangleq \max \{\widetilde{x}, \widehat{x}\}+\operatorname{ess} \sup B>0$ yields

$$
\begin{aligned}
u(\lambda x, \omega)=\widetilde{u}\left(\lambda\left[x-\frac{B(\omega)}{\lambda}\right]\right) & \leq \lambda^{\bar{\gamma}} \widetilde{u}\left(x-\frac{B(\omega)}{\lambda}\right)+\lambda^{\bar{\gamma}} C \\
& \leq \lambda^{\bar{\gamma}} \widetilde{u}(x-B(\omega))+\lambda^{\bar{\gamma}} K B(\omega)\left(1-\frac{1}{\lambda}\right)+\lambda^{\bar{\gamma}} C
\end{aligned}
$$

for all $\omega$ (outside a $\mathbb{P}$-null set), $\lambda \geq 1$ and $x \geq \bar{x}$. Note that $u^{+}(\bar{x}) \leq \tilde{u}(\bar{x})$ and the latter is deterministic. Hence, choosing $c \triangleq K \operatorname{ess} \sup B+C$ (which is constant, thus trivially integrable) gives the claimed result. We conclude by pointing out that any funcion $\widetilde{u}$ which is concave for sufficiently large $x$ satisfies the conditions $($ ii $),($ iii) above. 
We may now deduce the following auxiliary result, which provides an estimate for all $x \geq 0$, and not only for $x \geq \bar{x}$.

Lemma 2.11. Under Assumption 2.9 there is a random variable $C \geq 0$ a.s. such that $\mathbb{E}_{\mathbb{P}}[C]<+\infty$ and

$$
u^{+}(\lambda x) \leq \lambda^{\bar{\gamma}} u^{+}(x)+\lambda^{\bar{\gamma}} C
$$

for all $\lambda \geq 1$ and $x \geq 0$.

Proof. See Appendix A.

\section{Main results}

The optimal portfolio problem consists in choosing the "best" investment in the given assets: the one which maximises the expected utility from terminal wealth.

Definition 3.1. Let Assumption 2.6 be in force. Given any $x_{0} \geq 0$, the non-concave portfolio problem with initial wealth $x_{0}$ on a finite horizon $T$ is to find $\phi^{*} \in \psi\left(x_{0}\right)$ such that

$$
v^{*}\left(x_{0}\right) \triangleq \sup \left\{\mathbb{E}_{\mathbb{P}}\left[u\left(\Pi_{T}^{\phi}\right)\right]: \phi \in \Psi\left(x_{0}\right)\right\}=\mathbb{E}_{\mathbb{P}}\left[u\left(\Pi_{T}^{\phi^{*}}\right)\right] .
$$

We call $\phi^{*}$ an optimal strategy.

Remark 3.2. (i) Note that, due to Assumption 2.6, the expectations in (3.1) above exist, though they may be infinite. It is also immediate to check that, under Assumption 2.6, the strategy $\phi \equiv 0$ is in $\Psi\left(x_{0}\right)$ for all $x_{0} \geq 0$, so the supremum is taken over a nonempty set. In particular, $v^{*}\left(x_{0}\right) \geq \mathbb{E}_{\mathbb{P}}\left[u\left(x_{0}\right)\right]>-\infty$.

(ii) One may inquire why the existence of an optimal $\phi^{*}$ is important when the existence of $\varepsilon$-optimal strategies $\phi^{\varepsilon}$ (i.e., ones that are $\varepsilon$-close to the supremum over all strategies) is automatic, for all $\varepsilon>0$.

Firstly, non-existence of an optimal strategy $\phi^{*}$ usually means that an optimiser sequence $\left\{\phi^{1 / n} ; n \in \mathbb{N}\right\}$ shows a behaviour which is practically infeasible and counterintuitive (see Example 7.3 of Rásonyi and Stettner [18]).

Secondly, existence of $\phi^{*}$ normally goes together with some compactness property which would be needed for the construction of eventual numerical schemes to find the optimiser.

Here comes the main result of the present paper. It says that the optimisation problem (3.1) admits a solution.

Theorem 3.3. Let Assumptions 2.1, 2.6 and 2.9 hold true. Assume further that, for every $x_{0} \in[0,+\infty)$,

$$
v^{*}\left(x_{0}\right)<+\infty
$$

Then, for each $x_{0} \in[0,+\infty)$, there exists a strategy $\phi^{*}=\phi^{*}\left(x_{0}\right) \in \Psi\left(x_{0}\right)$ satisfying

$$
\mathbb{E}_{\mathbb{P}}\left[u\left(\Pi_{T}^{\phi^{*}}\right)\right]=v^{*}\left(x_{0}\right) .
$$

Proof. The proof will be given in Section 5, after appropriate preparations.

Remark 3.4. We would like to draw attention to the fact that, since Assumption 2.6 is in force, the well-posedness condition (WP) is actually equivalent to the apparently stronger one

$$
\sup _{\phi \in \Psi\left(x_{0}\right)} \mathbb{E}_{\mathbb{P}}\left[u^{+}\left(\Pi_{T}^{\phi}\right)\right]<+\infty .
$$

To see this, we recall that $\Pi_{T}^{\phi} \geq 0$ a.s. for every $\phi \in \Psi\left(x_{0}\right)$, hence

$$
\sup _{\phi \in \Psi\left(x_{0}\right)} \mathbb{E}_{\mathbb{P}}\left[u^{+}\left(\Pi_{T}^{\phi}\right)\right] \leq v^{*}\left(x_{0}\right)+\sup _{\phi \in \Psi\left(x_{0}\right)} \mathbb{E}_{\mathbb{P}}\left[u^{-}\left(\Pi_{T}^{\phi}\right)\right] \leq v^{*}\left(x_{0}\right)+\mathbb{E}_{\mathbb{P}}\left[u^{-}(0)\right]<+\infty
$$


As a very simple, yet important example to which the preceding theorem clearly applies, we mention the case of $u$ non-negative and bounded above and $S$ satisfying Assumption 2.1. Another relevant example is given by the following theorem. First, define

$$
\mathscr{W} \triangleq\left\{Y \in \Xi_{T}^{1}: \mathbb{E}_{\mathbb{P}}\left[|Y|^{p}\right]<+\infty \text { for all } p>0\right\} .
$$

Theorem 3.5. Let Assumptions 2.1, 2.6 and 2.9 hold true with $c, u^{+}(\bar{x}) \in \mathscr{W}$. Assume further that $\left\|\Delta S_{t}\right\|, 1 / \beta_{t} \in \mathscr{W}$ for every $t \in\{1, \ldots, T\}$, where the $\beta_{t}$ are the random variables figuring in Proposition 2.2. Then, for every $x_{0} \in[0,+\infty)$, condition (WP) is satisfied and there exists an optimal strategy $\phi^{*}=\phi^{*}\left(x_{0}\right) \in \mathscr{A}\left(x_{0}\right)$.

Proof. See Section 5.

\section{The one-step case}

In this section, we consider an $\mathscr{F}$-measurable function $Y: \Omega \rightarrow \mathbb{R}^{d}$, and a $\sigma$-algebra $\mathscr{G} \subseteq \mathscr{F}$ containing all $\mathbb{P}$-null sets of $\mathscr{F}$. This setting will be applied in the multi-step case (see the subsequent section) with $\mathscr{G}=\mathscr{F}_{t-1}$ and $Y=\Delta S_{t}$, for every fixed $t \in\{1, \ldots, T\}$.

Keeping in line with the notation of the previous section, we denote by $\Xi^{d}$ the family of all $\mathscr{G}$-measurable functions $\xi: \Omega \rightarrow \mathbb{R}^{d}$.

Moreover, let $\mathbb{P}^{Y \mid \mathscr{G}}: \mathscr{B}\left(\mathbb{R}^{d}\right) \times \Omega \rightarrow[0,1]$ be the unique (up to a set of measure zero) regular conditional distribution for $Y$ given $\mathscr{G}$. Now, for each $\omega \in \Omega$, let $\operatorname{supp}\left(\mathbb{P}^{Y \mid \mathscr{G}}(\cdot, \omega)\right)$ represent the support of $\mathbb{P}^{Y \mid \mathscr{G}}(\cdot, \omega)$ (which exists and is non-empty), and let $D(\omega)$ denote the affine hull of $\operatorname{supp}\left(\mathbb{P}^{Y \mid \mathscr{G}}(\cdot, \omega)\right)$, that is, $D(\omega) \triangleq \operatorname{aff}\left(\operatorname{supp}\left(\mathbb{P}^{Y \mid \mathscr{G}}(\cdot, \omega)\right)\right)$.

We shall also assume the following.

Assumption 4.1. For every $\omega \in \Omega, D(\omega)$ is a linear subspace of $\mathbb{R}^{d}$.

In addition, for every $\mathscr{G}$-measurable random variable $H: \Omega \rightarrow \mathbb{R}$ satisfying $H \geq 0$ a.s., define the set

$$
\Xi^{d}(H) \triangleq\left\{\xi \in \Xi^{d}:\langle\xi, Y\rangle \geq-H \text { a.s. }\right\} .
$$

Then in the particular case where $H=x$ a.s., for some $x \in[0,+\infty)$, we have

$$
\Xi^{d}(x) \triangleq\left\{\xi \in \Xi^{d}:\langle\xi, Y\rangle \geq-x \text { a.s. }\right\} \text {. }
$$

Finally, let $\widetilde{\Xi}^{d}$ denote the family of all functions $\xi \in \Xi^{d}$ such that $\xi(\omega) \in D(\omega)$ for each $\omega$. The notations $\tilde{\Xi}^{d}(H), \tilde{\Xi}^{d}(x)$ are self-explanatory.

We shall also impose the following condition, which can be regarded as absence of arbitrage (cf. Proposition 2.2).

Assumption 4.2. There exist $\mathscr{G}$-measurable random variables $\beta, \kappa>0$ a.s. such that

$$
\mathbb{P}(\langle\xi, Y\rangle \leq-\beta\|\xi\| \mid \mathscr{G}) \geq \kappa \text { a.s. on }\{D(\omega) \neq\{0\}\},
$$

for all $\xi \in \widetilde{\Xi}^{d}$. We may and will assume $\beta \leq 1$.

Assumption 4.3. Let the function $V:[0,+\infty) \times \Omega \rightarrow \mathbb{R}$ satisfy both properties below:

(i) for any fixed $x \in[0,+\infty)$, the function $V(x, \cdot): \Omega \rightarrow \mathbb{R}$ is measurable with respect to $\mathscr{F}$;

(ii) for every $\omega \in \Omega$, the function $V(\cdot, \omega):[0,+\infty) \rightarrow \mathbb{R}$ is continuous and non-decreasing.

We shall also need the following integrability conditions.

Assumption 4.4. For every $x \in[0,+\infty)$,

$$
\operatorname{ess}_{\xi \in \Xi^{d}(x)} \mathbb{E}_{\mathbb{P}}\left[V^{+}(x+\langle\xi(\cdot), Y(\cdot)\rangle, \cdot) \mid \mathscr{G}\right]<+\infty \text { a.s.. }
$$


Assumption 4.5. The conditional expectation of $V^{-}(0, \cdot): \Omega \rightarrow[0,+\infty)$ with respect to $\mathscr{G}$ is finite a.s., i.e.,

$$
\mathbb{E}_{\mathbb{P}}\left[V^{-}(0, \cdot) \mid \mathscr{G}\right]<+\infty \text { a.s. }
$$

Finally, we impose the following growth condition on $V$.

Assumption 4.6. There exist a constant $\gamma>0$ and a random variable $\bar{C}>0$ a.s. such that $\mathbb{E}_{\mathbb{P}}[\bar{C}]<+\infty$ and for each $\omega$,

$$
V^{+}(\lambda x, \omega) \leq \lambda^{\gamma} V^{+}(x, \omega)+\lambda^{\gamma} \bar{C}(\omega), \text { for all } \lambda \geq 1 \text { and for all } x \geq 0 .
$$

Next, we notice that denoting by $\hat{\xi}(\omega)$ the orthogonal projection of $\xi(\omega)$ on $D(\omega)$ for some $\xi \in \Xi^{d}$, we have $\hat{\xi} \in \Xi^{d}$ and $\langle\hat{\xi}, Y\rangle=\langle\xi, Y\rangle$ a.s., the reader is referred to Carassus and Rásonyi [5, Remark 8] for further details. This means that any portfolio can be replaced with its projection on $D$ without changing either its value or its desirability to the investor. We now recall that the set of all admissible strategies in $D$ is bounded.

Lemma 4.7. Given any $x_{0} \geq 0$, there exists a $\mathscr{G}$-measurable, real-valued random variable $K_{x_{0}}:=x_{0} / \beta \geq x_{0}$ such that, for every $x \in\left[0, x_{0}\right]$ and for every $\xi \in \tilde{\Xi}^{d}(x)$, we have

$$
\|\xi\| \leq K_{x_{0}} \text { a.s. }
$$

Proof. This is Lemma 2.1 in Rásonyi and Stettner [19].

As for the next lemma, it will allow us to apply the Fatou lemma to a sequence of conditional expectations tending to the essential supremum in (4.7) below.

Lemma 4.8. Given any $x \geq 0$, there is a non-negative random variable $L^{\prime}: \Omega \rightarrow \mathbb{R}$ such that $\mathbb{E}\left[L^{\prime} \mid \mathscr{G}\right]<+\infty$ a.s., and for every $\xi \in \tilde{\Xi}^{d}(x)$ the inequality

$$
V^{+}(x+\langle\xi(\cdot), Y(\cdot)\rangle, \cdot) \leq L_{x}
$$

holds a.s. for $L_{x}:=\left(x^{\bar{\gamma}}+1\right) L^{\prime}$.

Proof. See Appendix A.

Now a regular version of the essential supremum is shown to exist.

Lemma 4.9. There exists a function $G:[0,+\infty) \times \Omega \rightarrow \mathbb{R}$ satisfying the two properties below:

(i) the function $G(x, \cdot)$ is a version of $\operatorname{ess}^{\sup _{\xi \in \Xi^{d}(x)}} \mathbb{E}_{\mathbb{P}}[V(x+\langle\xi(\cdot), Y(\cdot)\rangle, \cdot) \mid \mathscr{G}]$ for each $x \in[0,+\infty)$;

(ii) for $\mathbb{P}$-a.e. $\omega \in \Omega$, the function $G(\cdot, \omega):[0,+\infty) \rightarrow \mathbb{R}$ is non-decreasing and continuous on $[0,+\infty)$.

Furthermore, given any $\mathscr{G}$-measurable random variable $H \geq 0$ a.s.,

$$
G(H(\cdot), \cdot)=\underset{\xi \in \Xi^{d}(H)}{\operatorname{ess} \sup _{1}} \mathbb{E}_{\mathbb{P}}[V(H(\cdot)+\langle\xi(\cdot), Y(\cdot)\rangle, \cdot) \mid \mathscr{G}] \text { a.s.. }
$$

Proof. See Appendix A.

Proposition 4.10. For any $\mathscr{G}$-measurable random variable $H \geq 0$ a.s. there exists $\widetilde{\xi}(H) \in$ $\tilde{\Xi}^{d}(H)$ with

$$
G(H(\cdot), \cdot)=\mathbb{E}_{\mathbb{P}}[V(H(\cdot)+\langle\widetilde{\xi}(H), Y(\cdot)\rangle, \cdot) \mid \mathscr{G}] \text { a.s.. }
$$

Proof. See Appendix A. 


\section{The multi-step case}

In this section, we shall follow Carassus and Rásonyi [5]; Rásonyi and Stettner [18, 19], and employ a dynamic programming approach to split the original optimisation problem into a number of sub-problems at different trading dates. Our goal is to invoke the results of the preceding section, thus allowing us to obtain an optimal solution at each stage. Combining them in an appropriate way will yield a globally optimal investment strategy.

Proof of Theorem 3.3. We must prove that some crucial assumptions of Section 4 are preserved at each time step. So let us start by defining

$$
U_{T}(x, \omega) \triangleq u(x, \omega), \quad x \geq 0, \omega \in \Omega .
$$

We wish to apply the results of Section 4 with $Y \triangleq \Delta S_{T}, \mathscr{G} \triangleq \mathscr{F}_{T-1}$ and $V \triangleq U_{T}$.

(i) Since Assumption 2.1 holds by hypothesis, Theorem 3 in Jacod and Shiryaev [10] implies that the affine space $D_{T}(\omega)$ is a linear subspace of $\mathbb{R}^{d}$ a.s., therefore Assumption 4.1 is verified (after trivial adjustments). It follows from Proposition 2.2 that Assumption 4.2 holds as well.

(ii) We note further that Assumption 4.3 is also true. Indeed, if we fix any $x \geq 0$, then it follows immediately from the definition of a random utility that the function $U_{T}(x, \cdot): \Omega \rightarrow \mathbb{R}$ is $\mathscr{F}_{T}$-measurable and, for all $\omega, U_{T}(\omega, \cdot)$ is continuous and nondecreasing on $[0,+\infty)$.

(iii) We now claim that Assumption 4.4 is satisfied. In order to do so, fix an arbitrary $x \geq 0$. It can be easily shown that

$$
\left\{\mathbb{E}_{\mathbb{P}}\left[U_{T}^{+}\left(x+\left\langle\xi(\cdot), \Delta S_{T}(\cdot)\right\rangle, \cdot\right) \mid \mathscr{F}_{T-1}\right] ; \xi \in \Xi_{T-1}^{d}(x)\right\}
$$

is directed upwards, so we can find a countable sequence of random vectors $\left\{\xi_{n} ; n \in \mathbb{N}\right\} \subseteq$ $\Xi^{d}(x)$ attaining the essential supremum, i.e., such that

$$
\begin{aligned}
\lim _{n \rightarrow+\infty} \mathbb{E}_{\mathbb{P}}\left[U _ { T } ^ { + } \left(x+\left\langle\xi_{n}(\cdot),\right.\right.\right. & \left.\left.\left.\Delta S_{T}(\cdot)\right\rangle, \cdot\right) \mid \mathscr{F}_{T-1}\right] \\
& =\operatorname{ess}_{\xi \in \Xi_{T-1}^{d}(x)} \mathbb{E}_{\mathbb{P}}\left[U_{T}^{+}\left(x+\left\langle\xi(\cdot), \Delta S_{T}(\cdot)\right\rangle, \cdot\right) \mid \mathscr{F}_{T-1}\right] \text { a.s. }
\end{aligned}
$$

in a non-decreasing way. Therefore, it follows from the Monotone Convergence Theorem and from the definition of the conditional expectation that

$$
\begin{aligned}
\mathbb{E}_{\mathbb{P}}\left[\operatorname { e s s } _ { \xi \in \Xi _ { T - 1 } ^ { d } ( x ) } \mathbb { E } _ { \mathbb { P } } \left[U_{T}^{+}(x+\right.\right. & \left.\left.\left.\left\langle\xi(\cdot), \Delta S_{T}(\cdot)\right\rangle, \cdot\right) \mid \mathscr{F}_{T-1}\right]\right] \\
& =\lim _{n \rightarrow+\infty} \mathbb{E}_{\mathbb{P}}\left[\mathbb{E}_{\mathbb{P}}\left[U_{T}^{+}\left(x+\left\langle\xi_{n}(\cdot), \Delta S_{T}(\cdot)\right\rangle, \cdot\right) \mid \mathscr{F}_{T-1}\right]\right] \\
& =\sup _{n \in \mathbb{N}} \mathbb{E}_{\mathbb{P}}\left[U_{T}^{+}\left(x+\left\langle\xi_{n}(\cdot), \Delta S_{T}(\cdot)\right\rangle, \cdot\right)\right] .
\end{aligned}
$$

Now, it is straightforward to check that, given any $\xi \in \Xi^{d}(x)$, the $\mathbb{R}^{d}$-valued process defined by

$$
\left(\phi_{\xi}\right)_{t} \triangleq \begin{cases}\xi, & \text { if } t=T \\ 0, & \text { otherwise }\end{cases}
$$

is a portfolio in $\Psi(x)$, with

$$
\begin{aligned}
\mathbb{E}_{\mathbb{P}}\left[u^{+}\left(\Pi_{T}^{\bar{\phi}_{\xi}}(\cdot), \cdot\right) \mid \mathscr{F}_{T-1}\right] & =\mathbb{E}_{\mathbb{P}}\left[u^{+}\left(x+\left\langle\xi(\cdot), \Delta S_{T}(\cdot)\right\rangle, \cdot\right) \mid \mathscr{F}_{T-1}\right] \\
& =\mathbb{E}_{\mathbb{P}}\left[U_{T}^{+}\left(x+\left\langle\xi(\cdot), \Delta S_{T}(\cdot)\right\rangle, \cdot\right) \mid \mathscr{F}_{T-1}\right] \text { a.s. }
\end{aligned}
$$


In particular, the preceding equality and condition (WP) imply that

$$
\mathbb{E}_{\mathbb{P}}\left[\mathbb{E}_{\mathbb{P}}\left[U_{T}^{+}\left(x+\left\langle\xi(\cdot), \Delta S_{T}(\cdot)\right\rangle, \cdot\right) \mid \mathscr{F}_{T-1}\right]\right]=\mathbb{E}_{\mathbb{P}}\left[u^{+}\left(\Pi_{T}^{\bar{\phi}_{\xi}}(\cdot), \cdot\right)\right]<+\infty,
$$

and so $\mathbb{E}_{\mathbb{P}}\left[U_{T}^{+}\left(x+\left\langle\xi(\cdot), \Delta S_{T}(\cdot)\right\rangle, \cdot\right) \mid \mathscr{F}_{T-1}\right]<+\infty$ a.s. (thus, the conditional expectation is well-defined and finite a.s.).

At last, setting $\bar{\phi}_{n} \triangleq \bar{\phi}_{\xi_{n}}$, combining the results obtained above and invoking hypothesis (WP) once again, we conclude that

$$
\begin{aligned}
\mathbb{E}_{\mathbb{P}}\left[\operatorname{esssup}_{\xi \in \Xi_{T-1}^{d}(x)} \mathbb{E}_{\mathbb{P}}\left[U_{T}^{+}\left(x+\left\langle\xi(\cdot), \Delta S_{T}(\cdot)\right\rangle, \cdot\right) \mid \mathscr{F}_{T-1}\right]\right] \\
=\sup _{n \in \mathbb{N}} \mathbb{E}_{\mathbb{P}}\left[u^{+}\left(\Pi_{T}^{\phi_{n}}(\cdot), \cdot\right)\right]<+\infty,
\end{aligned}
$$

hence $\operatorname{ess} \sup _{\xi \in \Xi_{T-1}^{d}(x)} \mathbb{E}_{\mathbb{P}}\left[U_{T}^{+}\left(x+\left\langle\xi(\cdot), \Delta S_{T}(\cdot)\right\rangle, \cdot\right) \mid \mathscr{F}_{T-1}\right]<+\infty$ a.s..

(iv) The next step is to show that we have Assumption 4.5 as well. In fact, due to Assumption 2.6, it is immediate that

$$
\mathbb{E}_{\mathbb{P}}\left[\mathbb{E}_{\mathbb{P}}\left[U_{T}^{-}(0, \cdot) \mid \mathscr{F}_{T-1}\right]\right]=\mathbb{E}_{\mathbb{P}}\left[U_{T}^{-}(0, \cdot)\right]=\mathbb{E}_{\mathbb{P}}\left[u^{-}(0, \cdot)\right]<+\infty,
$$

so $\mathbb{E}_{\mathbb{P}}\left[U_{T}^{-}(0, \cdot) \mid \mathscr{F}_{T-1}\right]<+\infty$ a.s..

(v) Lastly, let the constant $\bar{\gamma}>0$ and the integrable random variable $C>0$ be those given by Assumption 2.9 and Lemma 2.11, respectively. Then, for every $\omega \in \Omega$, we obtain

$$
U_{T}^{+}(\lambda x, \omega)=u^{+}(\lambda x, \omega) \leq \lambda^{\bar{\gamma}} u^{+}(x, \omega)+C \lambda^{\bar{\gamma}}=\lambda^{\bar{\gamma}} U_{T}^{+}(x, \omega)+C \lambda^{\bar{\gamma}},
$$

for all $\lambda \geq 1$ and $x \geq 0$.

Hence, by Lemma 4.9, there exists a function $G_{T-1}:[0,+\infty) \times \Omega \rightarrow \mathbb{R}$ such that, for every $\omega$ in a $\mathbb{P}$-full measure set, the function $G_{T-1}(\cdot, \omega):[0,+\infty) \rightarrow \mathbb{R}$ is non-decreasing and continuous on $[0,+\infty)$. Moreover, for every $x \in[0,+\infty)$,

$$
G_{T-1}(x, \cdot)=\underset{\xi \in \Xi_{T-1}^{d}(x)}{\operatorname{ess} \sup _{\mathbb{P}}} \mathbb{E}_{\mathbb{P}}\left[U_{T}\left(x+\left\langle\xi(\cdot), \Delta S_{T}(\cdot)\right\rangle, \cdot\right) \mid \mathscr{F}_{T-1}\right] \text { a.s.. }
$$

In addition, Proposition 4.10 gives us, for each $H \in \Xi_{T-1}^{1}$, an $\mathscr{F}_{T-1}$-measurable function $\widetilde{\xi}_{T}(H): \Omega \rightarrow \mathbb{R}^{d}$ such that

$$
\begin{aligned}
G_{T-1}(H)=\operatorname{ess}_{\xi \in \Xi_{T-1}^{d}(H)} \mathbb{E}_{\mathbb{P}}\left[U_{T}(H\right. & \left.\left.+\left\langle\xi(\cdot), \Delta S_{T}(\cdot)\right\rangle, \cdot\right) \mid \mathscr{F}_{T-1}\right] \\
& =\mathbb{E}_{\mathbb{P}}\left[U_{T}\left(H+\left\langle\widetilde{\xi}_{T}(H)(x, \cdot), \Delta S_{T}(\cdot)\right\rangle, \cdot\right) \mid \mathscr{F}_{T-1}\right] \text { a.s.. }
\end{aligned}
$$

Let us now proceed to the next stage of dynamic programming. Let $U_{T-1}:[0,+\infty) \times$ $\Omega \rightarrow \mathbb{R}$ be the function given by $U_{T-1}(x, \omega) \triangleq G_{T-1}(x, \omega)$. As before, we would like to use the results of Section 4 , this time with $Y \triangleq \Delta S_{T-1}, \mathscr{G} \triangleq \mathscr{F}_{T-2}$ and $V \triangleq U_{T-1}$.

(i) That Assumption 4.1 and Assumption 4.2 are both true follows as before.

(ii) Next, we prove that Assumption 4.3 holds. In fact, given any $x \geq 0$, the function $U_{T-1}(x, \cdot): \Omega \rightarrow \mathbb{R}$ is $\mathscr{F}_{T-1}$-measurable. On the other hand, for a.e. every $\omega$, we have by definition of $U_{T-1}$ that $U_{T-1}(\cdot, \omega)$ is a non-decreasing continuous function on $[0,+\infty)$. 
(iii) We show that we also have Assumption 4.4. Indeed, letting $x \geq 0$ be arbitrary, but fixed, it can be easily checked, in the same way as before (the construction of the portfolio becoming more involved, but totally analogous), that for every $\xi \in \Xi_{T-2}^{d}(x)$, the conditional expectation

$$
\mathbb{E}_{\mathbb{P}}\left[U_{T-1}\left(x+\left\langle\xi(\cdot), \Delta S_{T-1}(\cdot)\right\rangle, \cdot\right) \mid \mathscr{F}_{T-2}\right]
$$

is not only well-defined, but also finite a.s.. Furthermore,

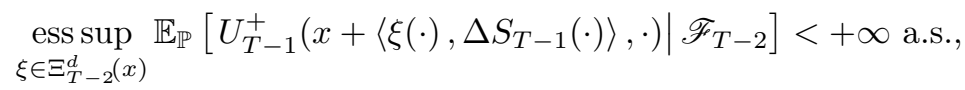

as desired.

(iv) We proceed with the proof that Assumption 4.5 is also verified. Given any $x \geq 0$, it is clear that

$$
U_{T-1}(x, \cdot)=G_{T-1}(x, \cdot) \geq \mathbb{E}_{\mathbb{P}}\left[U_{T}(x, \cdot) \mid \mathscr{F}_{T-1}\right] \text { a.s. },
$$

where the inequality is due to $0 \in \Xi_{T-1}^{d}(x)$ and to the definition of the essential supremum, thus in particular we can use Jensen's inequality (for the conditional expectation) to obtain

$$
\begin{aligned}
\mathbb{E}_{\mathbb{P}}\left[\mathbb{E}_{\mathbb{P}}\left[U_{T-1}^{-}(0, \cdot) \mid \mathscr{F}_{T-2}\right]\right] & =\mathbb{E}_{\mathbb{P}}\left[U_{T-1}^{-}(0, \cdot)\right] \\
& \leq \mathbb{E}_{\mathbb{P}}\left[\mathbb{E}_{\mathbb{P}}\left[U_{T}^{-}(0, \cdot) \mid \mathscr{F}_{T-1}\right]\right]=\mathbb{E}_{\mathbb{P}}\left[U_{T}^{-}(0, \cdot)\right]=\mathbb{E}_{\mathbb{P}}\left[u^{-}(0, \cdot)\right]
\end{aligned}
$$

which in turn implies (recall Assumption 2.6) that $\mathbb{E}_{\mathbb{P}}\left[U_{T-1}^{-}(0, \cdot) \mid \mathscr{F}_{T-2}\right]<+\infty$ a.s..

(v) We finish by noting that, taking again $\bar{\gamma}>0$ and $C>0$ to be, respectively, the real numbers of Assumption 2.9 and Lemma 2.11, we have that, for every $\lambda \geq 1$ and $x \geq 0$,

$$
\begin{aligned}
U_{T-1}^{+}(\lambda x, \cdot) & \leq \mathbb{E}_{\mathbb{P}}\left[U_{T}^{+}\left(\lambda x+\left\langle\widetilde{\xi}_{T}(\lambda x), \Delta S_{T}(\cdot)\right\rangle, \cdot\right) \mid \mathscr{F}_{T-1}\right] \\
& \leq \lambda^{\bar{\gamma}} \mathbb{E}_{\mathbb{P}}\left[U_{T}\left(x+\left\langle\widetilde{\xi}_{T}(\lambda x) / \lambda, \Delta S_{T}(\cdot)\right\rangle, \cdot\right) \mid \mathscr{F}_{T-1}\right]+\lambda^{\bar{\gamma}} \mathbb{E}_{\mathbb{P}}\left[C \mid \mathcal{F}_{T-1}\right] \text { a.s. },
\end{aligned}
$$

where the first inequality follows from the conditional Jensen inequality, and the second one uses (5.1). It is easy to see that $\widetilde{\xi}_{T}(\lambda x) / \lambda \in \Xi_{T-1}^{d}(x)$ and we conclude that, for every $\lambda \geq 1$ and $x \geq 0, U_{T-1}^{+}(\lambda x, \cdot) \leq \lambda^{\bar{\gamma}} U_{T-1}^{+}(x, \cdot)+\lambda^{\bar{\gamma}} \bar{C}$ a.s. for $\bar{C}:=\mathbb{E}_{\mathbb{P}}\left[C \mid \mathcal{F}_{T-1}\right]$.

Using the regularity of the paths of $U_{T-1}$, we get that, for a.e. $\omega$ the inequality $U_{T-1}^{+}(\lambda x, \omega) \leq \lambda^{\bar{\gamma}} U_{T-1}^{+}(x, \omega)+\lambda^{\bar{\gamma}} \bar{C}$ holds for all $\lambda \geq 1$ and $x \geq 0$, so Assumption 4.6 is verified.

Consequently, we can apply Lemma 4.9 and Proposition 4.10 to obtain functions $G_{T-2}$ and $\widetilde{\xi}_{T-1}$ satisfying the desired properties. Proceeding in a similar way for the remaining values of $t \in\{T-2, \ldots, 1\}$, we construct the functions $U_{T-2}, \ldots, U_{1}, U_{0}$ and $\widetilde{\xi}_{T-2}, \ldots, \widetilde{\xi}_{1}$.

We then inductively define $\Pi_{0}^{\phi^{*}}=x_{0}, \phi_{1}^{*}=\tilde{\xi}_{1}\left(x_{0}\right), \phi_{t}^{*}=\tilde{\xi}_{t}\left(\Pi_{t-1}^{\phi^{*}}\right)$. The remainder of the proof, showing optimality of $\phi^{*}$, unfolds exactly as the proof of Proposition 3.2 in Rásonyi and Stettner [19].

Proof of Theorem 3.5. We will prove by backward induction that $U_{t}(x) \leq J_{t}\left[x^{\bar{\gamma}}+1\right]$ for some $J_{t} \in \mathscr{W}$, for all $t$. Assumption 2.9 shows that, for all $x \geq \bar{x}$,

$$
U_{T}(x)=u(x) \leq(x / \bar{x})^{\bar{\gamma}}\left[u^{+}(\bar{x})+c\right],
$$

and for $0 \leq x<\bar{x}$

$$
U_{T}(x) \leq u^{+}(\bar{x})
$$

so we can set

$$
J_{T}:=\max \left\{\left[u^{+}(\bar{x})+c\right] / \bar{x}^{\bar{\gamma}}, u^{+}(\bar{x})\right\}
$$


and the latter is clearly in $\mathscr{W}$ by our assumptions. Let us assume that the statement has been shown for $t+1, \ldots, T$. Estimate, using Lemma 4.7,

$$
\begin{aligned}
& U_{t}(x)=\text { ess. } \sup _{\xi \in \tilde{\Xi}_{t}^{d}(x)} \mathbb{E}_{\mathbb{P}}\left[U_{t+1}\left(x+\left\langle\xi, \Delta S_{t+1}\right\rangle\right) \mid \mathscr{F}_{t}\right] \leq \\
& \text { ess. } \sup _{\xi \in \tilde{\Xi}_{t}^{d}(x)} \mathbb{E}_{\mathbb{P}}\left[J_{t+1}\left(x+\|\xi\| \| \Delta S_{t+1}||\right)^{\gamma}+J_{t+1} \mid \mathscr{F}_{t}\right] \leq \\
& x^{\bar{\gamma}^{\gamma}} \mathbb{E}_{\mathbb{P}}\left[J_{t+1}\left(1+\| \Delta S_{t+1}|| / \beta_{t+1}\right)^{\bar{\gamma}} \mid \mathscr{F}_{t}\right]+\mathbb{E}_{\mathbb{P}}\left[J_{t+1} \mid \mathscr{F}_{t}\right],
\end{aligned}
$$

so we may set $J_{t}:=\mathbb{E}_{\mathbb{P}}\left[J_{t+1}\left(1+|| \Delta S_{t+1}|| / \beta_{t+1}\right)^{\bar{\gamma}} \mid \mathscr{F}_{t}\right] \in \mathscr{W}$. It follows that $v^{*}(x) \leq E U_{0}(x) \leq$ $\left(1+x^{\bar{\gamma}}\right) E J_{0}<\infty$ and we can conclude using Theorem 3.3.

\section{A Appendix: Proofs and auxiliary results}

Except where explicitly stated otherwise, we stay in the setting of Section 4.

Example A.1. It is not difficult to find utilities which are bounded above and yet have nonzero (actually, infinite) asymptotic elasticity, as the example below shows (the construction being inspired by the proof of Lemma 6.5 in Kramkov and Schachermayer [14]).

In fact, let $f:[0,+\infty) \rightarrow \mathbb{R}$ be the continuous and strictly increasing function which takes the values

$$
\begin{gathered}
f(n) \triangleq \frac{1}{2}-\frac{1}{n+1}=\frac{n-1}{2(n+1)}, \\
f\left(n+1 / 2-a_{n}\right) \triangleq f(n)+a_{n}, \\
f\left(n+1 / 2+a_{n}\right) \triangleq f(n+1)-a_{n},
\end{gathered}
$$

with $a_{n} \triangleq 1 /(4(n+1)(n+2))$, and which is linear between the points where it has been defined.

Clearly $f(+\infty)=1 / 2$ and $f(1)=0$. We also note that $f(0)=-1 / 2>-\infty$. Moreover, the piecewise linearity of $f$ and trivial computations yield

$$
f^{\prime}(x)=\frac{f\left(n+1 / 2+a_{n}\right)-f\left(n+1 / 2-a_{n}\right)}{2 a_{n}}=1
$$

for any $x \in\left(n+1 / 2-a_{n}, n+1 / 2+a_{n}\right)$, so in particular $f^{\prime}(n+1 / 2)$ equals 1 . Furthermore, we have the following inequality,

$$
\frac{f(n+1 / 2)}{n+1 / 2} \leq \frac{f(n+1)}{n+1 / 2}=\frac{n}{(n+2)(2 n+1)},
$$

thus combining all of the above gives $\lim _{n \rightarrow+\infty}(n+1 / 2) f^{\prime}(n+1 / 2) / f(n+1 / 2)=+\infty$, and hence $A E_{+}(u)=+\infty$. We finish by noticing that, as in the proof of Lemma 6.5 in Kramkov and Schachermayer [14], $f$ can be slightly modified in such a way that it becomes smooth and our conclusion is still valid.

Proof of Lemma 2.11. Let us begin by noticing that the inequality is trivial if $u(\lambda x)<0$ so we may and will assume $u(\lambda x)=u^{+}(\lambda x)$. Consider an arbitrary $x \in[0, \bar{x})$. Then we can use the fact that $u$ is non-decreasing and inequality (2.4), to obtain $u^{+}(\lambda x) \leq u^{+}(\lambda \bar{x})=$ $u(\lambda \bar{x}) \leq \lambda^{\bar{\gamma}} u(\bar{x})+\lambda^{\bar{\gamma}} c$ for any $\lambda \geq 1$.

On the other hand, for every $x \geq \bar{x}$, we have $u^{+}(\lambda x)=u(\lambda x) \leq \lambda^{\bar{\gamma}}[u(x)+c] \leq \lambda^{\bar{\gamma}}\left[u^{+}(x)+\right.$ $c]$ for all $\lambda \geq 1$.

Hence, choosing $C \triangleq u^{+}(\bar{x})+c \geq 0$, and combining the two previous inequalities yields

$$
u^{+}(\lambda x) \leq \max \left\{\lambda^{\bar{\gamma}}[u(\bar{x})+c], \lambda^{\bar{\gamma}}\left[u^{+}(x)+c\right]\right\} \leq \lambda^{\bar{\gamma}}\left[u^{+}(x)+u^{+}(\bar{x})+c\right]=\lambda^{\bar{\gamma}} u^{+}(x)+\lambda^{\bar{\gamma}} C
$$

for all $\lambda \geq 1$ and for all $x \geq 0$, as claimed. Lastly, note that $\mathbb{E}_{\mathbb{P}}[C]<+\infty$ since $\mathbb{E}_{\mathbb{P}}\left[u^{+}(\bar{x})\right]<$ $+\infty$. 
Proof of Lemma 4.8. Let $\Theta$ be the set of functions from $\{1, \ldots, d\}$ to $\{-\sqrt{d}, \sqrt{d}\}$. Let $x>0$. Then we have by 4.7 , for all $\xi \in \tilde{\Xi}^{d}(x)$,

$$
x+\langle\xi, Y\rangle \leq x(1+\|Y\| / \beta) \leq x\left(1+(1 / \beta) \max _{\tau \in \Theta}\langle\tau, Y\rangle\right)
$$

hence

$$
V^{+}(x+\langle\xi, Y\rangle) \leq V^{+}\left(x\left(1+(1 / \beta) \max _{\tau \in \Theta}\langle\tau, Y\rangle\right) \leq \sum_{\tau \in \Theta} V^{+}(x(1+\langle\tau / \beta, Y\rangle))\right.
$$

a.s. Just like in the proof of Lemma 2.3 in Rásonyi and Stettner [19], one can show the existence of $g \in \Xi^{d}(x)$ and $\varepsilon_{\tau} \in \Xi^{1}, \varepsilon_{\tau} \in(0,1)$ such that $\tilde{g}_{\tau}:=g+\varepsilon_{\tau}(\tau / \beta-g) \in \Xi^{d}(x)$. It follows that, for $x \leq 1$,

$$
V^{+}(x+\langle\xi, Y\rangle) \leq \sum_{\tau \in \Theta} V^{+}(1+\langle\tau / \beta, Y\rangle)
$$

and for $x>1$,

$$
V^{+}(x+\langle\xi, Y\rangle) \leq \sum_{\tau \in \Theta} x^{\bar{\gamma}}\left(V^{+}(1+\langle\tau / \beta, Y\rangle)+\bar{C}\right)
$$

by Assumption 4.6. Applying the same assumption again, we get

$$
\begin{aligned}
V^{+}\left(1+\left\langle\frac{\tau}{\beta}, Y\right\rangle\right) & \leq \frac{1}{\varepsilon_{\tau} \bar{\gamma}}\left(V^{+}\left(\varepsilon_{\tau}\left(1+\left\langle\frac{\tau}{\beta}, Y\right\rangle\right)\right)+\bar{C}\right) \\
& \leq \frac{1}{\varepsilon_{\tau} \bar{\gamma}}\left(V^{+}\left(\varepsilon_{\tau}(1+\langle g, Y\rangle)+\varepsilon_{\tau}\left\langle\left(\frac{\tau}{\beta}-g\right), Y\right\rangle\right)+\bar{C}\right) \\
& \leq \frac{1}{\varepsilon_{\tau} \bar{\gamma}}\left(V^{+}\left(1+\langle g, Y\rangle+\varepsilon_{\tau}\left\langle\left(\frac{\tau}{\beta}-g\right), Y\right\rangle\right)+\bar{C}\right) \\
& =\frac{1}{\varepsilon_{\tau} \bar{\gamma}}\left(V^{+}\left(1+\left\langle\tilde{g}_{\tau}, Y\right\rangle\right)+\bar{C}\right)
\end{aligned}
$$

where the last inequality holds true since $1+\langle g, Y\rangle \geq 0$ a.s. Now let

$$
L^{\prime}=\sum_{\tau \in \Theta}\left(\frac{1}{\varepsilon_{\tau} \bar{\gamma}}\left(V^{+}\left(1+\left\langle\tilde{g}_{\tau}, Y\right\rangle\right)+\bar{C}\right)+\bar{C}\right)+V^{+}(0)
$$

where the last term is added to cover the case $x=0$ as well. By assumption, $\mathbb{E}_{\mathbb{P}}[\bar{C} \mid \mathscr{G}]<\infty$ and by Assumption 4.4 we have $\mathbb{E}_{\mathbb{P}}\left[V^{+}\left(1+\tilde{g}_{\tau} Y\right) \mid \mathscr{G}\right]<\infty$, so the proof is completed.

Proof of Lemma 4.9. Let us first choose, for each positive rational number $q$, a version $F(q, \omega)$ of $\operatorname{ess}_{\sup _{\xi \in \Xi d}(q)} \mathbb{E}_{\mathbb{P}}[V(q+\langle\xi(\cdot), Y(\cdot)\rangle, \cdot) \mid \mathscr{G}]$.

Let us specify, for each $x \in[0,+\infty)$,

$$
G(x, \omega) \triangleq \inf _{\substack{q \in \mathbb{Q} \\ q>x}} F(q, \omega) .
$$

Clearly, when $x \geq 0$ is in $\mathbb{Q}$ then $G(x, \cdot) \geq F(x, \cdot)$ a.s. In addition, for each $x \geq 0$ we have that $G(x, \cdot)$ is $\mathscr{G}$-measurable. We shall split the remainder of the proof into five separate parts.

(i) With the above definition, it is straightforward to check that, for a.e. $\omega \in \Omega$, the function $G(\cdot, \omega)$ is non-decreasing. It is also clear that, for every $\omega$ outside a $\mathbb{P}$-null set, $G(x, \omega)<+\infty$ for all $x \geq 0$. 
(ii) We proceed to show that, for all $x \in[0,+\infty)$,

$$
G(x, \cdot)=\underset{\xi \in \Xi^{d}(x)}{\operatorname{ess} \sup _{\mathbb{P}}} \mathbb{E}_{\mathbb{P}}[V(x+\langle\xi(\cdot), Y(\cdot)\rangle, \cdot) \mid \mathscr{G}] \text { a.s.. }
$$

In order to do so, let us fix an arbitrary $x \in[0,+\infty)$. Then, for every $q \in \mathbb{Q}, q>x$, the inequality

$$
\underset{\xi \in \Xi^{d}(x)}{\operatorname{ess} \sup } \mathbb{E}_{\mathbb{P}}[V(x+\langle\xi(\cdot), Y(\cdot)\rangle, \cdot) \mid \mathscr{G}] \leq F(q, \cdot)
$$

holds a.s., thus we get a.s.

$$
\underset{\xi \in \Xi^{d}(x)}{\operatorname{ess} \sup _{\mathbb{P}}} \mathbb{E}_{\mathbb{P}}[V(x+\langle\xi(\cdot), Y(\cdot)\rangle, \cdot) \mid \mathscr{G}](\omega) \leq G(x, \omega) .
$$

It remains to verify that the reverse inequality is also true (except possibly on a set of measure zero). This will be achieved in three steps.

(a) Let us start by taking a strictly decreasing sequence $\left\{q_{n} ; n \in \mathbb{N}\right\}$ of rational numbers satisfying $x<q_{n}<x+1$ and $\lim _{n \rightarrow+\infty} q_{n}=x$. Now, given any $n \in \mathbb{N}$, it is straightforward that the family $\left\{\mathbb{E}_{\mathbb{P}}\left[V\left(q_{n}+\langle\xi(\cdot), Y(\cdot)\rangle, \cdot\right) \mid \mathscr{G}\right] ; \xi \in \Xi^{d}\left(q_{n}\right)\right\}$ is directed upwards, therefore one can find $\zeta_{n} \in \Xi^{d}\left(q_{n}\right)$ such that

$$
\mathbb{E}_{\mathbb{P}}\left[V\left(q_{n}+\left\langle\zeta_{n}(\cdot), Y(\cdot)\right\rangle, \cdot\right) \mid \mathscr{G}\right](\omega) \geq F\left(q_{n}, \omega\right)-\frac{1}{n} \text { a.s. }
$$

(b) Next, fix an arbitrary $n$. It was observed above that $\zeta_{n} \in \Xi^{d}\left(q_{n}\right) \subseteq \Xi^{d}(x+1)$. Thus, taking $\widehat{\zeta}_{n}$ to be its projection on $D$, we know that

$$
\begin{aligned}
\mathbb{E}_{\mathbb{P}}\left[V\left(q_{n}+\left\langle\widehat{\zeta}_{n}(\cdot), Y(\cdot)\right\rangle, \cdot\right) \mid \mathscr{G}\right] & =\mathbb{E}_{\mathbb{P}}\left[V\left(q_{n}+\left\langle\zeta_{n}(\cdot), Y(\cdot)\right\rangle, \cdot\right) \mid \mathscr{G}\right] \\
& \geq F\left(q_{n}, \cdot\right)-\frac{1}{n} \text { a.s.. }
\end{aligned}
$$

Moreover, Lemma 4.7 allows us to conclude that $\left\|\widehat{\zeta}_{n}\right\|_{\mathbb{R}^{n}} \leq K_{x+1}$ a.s.. Therefore, we can extract a random subsequence $\left\{\widehat{\zeta}_{n_{k}} ; k \in \mathbb{N}\right\}$ such that $\lim _{k \rightarrow+\infty} \widehat{\zeta}_{n_{k}}=\zeta$ a.s., for some $\mathscr{G}$-measurable random variable $\zeta$. But then

$$
x+\langle\zeta(\omega), Y(\omega)\rangle=\lim _{k \rightarrow+\infty}\left(q_{n_{k}(\omega)}+\left\langle\widehat{\zeta}_{n_{k}(\omega)}(\omega), Y(\omega)\right\rangle\right) \geq 0
$$

for $\mathbb{P}$-a.e. $\omega \in \Omega$, i.e. $\zeta \in \Xi^{d}(x)$, which in turn implies that

$$
\underset{\xi \in \Xi^{d}(x)}{\operatorname{ess} \sup _{\mathbb{P}}} \mathbb{E}_{\mathbb{P}}[V(x+\langle\xi(\cdot), Y(\cdot)\rangle, \cdot) \mid \mathscr{G}] \geq \mathbb{E}_{\mathbb{P}}[V(x+\langle\zeta(\cdot), Y(\cdot)\rangle, \cdot) \mid \mathscr{G}] \text { a.s.. }
$$

(c) Finally, let us define the random variables $f_{k}: \Omega \rightarrow \mathbb{R}$ as follows,

$$
f_{k}(\omega) \triangleq V\left(q_{n_{k}(\omega)}+\left\langle\widehat{\zeta}_{n_{k}(\omega)}(\omega), Y(\omega)\right\rangle, \omega\right), \quad \omega \in \Omega .
$$

By virtue of the way the sequence $\left\{q_{n} ; n \in \mathbb{N}\right\}$ and the random subsequence $\left\{\widehat{\zeta}_{n_{k}} ; k \in \mathbb{N}\right\}$ were produced, and of the continuity of the paths of $V$ (see Assumption 4.3), it is clear that $\lim _{k \rightarrow+\infty} f_{k}=V(x+\langle\zeta(\cdot), Y(\cdot)\rangle, \cdot)$ a.s.. We further observe that, for $\mathbb{P}$-a.e. $\omega \in \Omega$

$$
f_{k}(\omega) \leq V\left(x+1+\left\langle\widehat{\zeta}_{n_{k}(\omega)}(\omega), Y(\omega)\right\rangle, \omega\right) \leq\left(x^{\bar{\gamma}}+1\right) L^{\prime}(\omega),
$$


where the first inequality follows from the monotonicity of $V$ (again we refer to Assumption 4.3), and the second inequality is a simple consequence of Lemma 4.8 combined with the fact that

$$
x+1+\left\langle\widehat{\zeta}_{n_{k}(\omega)}(\omega), Y(\omega)\right\rangle \geq q_{n_{k}(\omega)}+\left\langle\widehat{\zeta}_{n_{k}(\omega)}(\omega), Y(\omega)\right\rangle \geq 0
$$

a.s. Hence, we may apply the Fatou lemma to conclude that

$$
\begin{aligned}
\mathbb{E}_{\mathbb{P}}[V(x+\langle\zeta(\cdot), Y(\cdot)\rangle, \cdot) \mid \mathscr{G}] & \geq \limsup _{k \rightarrow+\infty} \mathbb{E}_{\mathbb{P}}\left[f_{k} \mid \mathscr{G}\right] \\
\geq & \liminf _{k \rightarrow+\infty} F\left(q_{n_{k}(\cdot)}, \cdot\right)-\frac{1}{n_{k}} \geq \inf _{n \in \mathbb{N}} F\left(q_{n}, \cdot\right) \text { a.s. }
\end{aligned}
$$

Combining equations (A.2) and (A.3) finally gives the intended inequality

$$
\operatorname{ess~sup}_{\xi \in \Xi d(x)} \mathbb{E}_{\mathbb{P}}[V(x+\langle\xi(\cdot), Y(\cdot)\rangle, \cdot) \mid \mathscr{G}] \geq \inf _{n \in \mathbb{N}} F\left(q_{n}, \cdot\right) \geq G(x, \cdot) \text { a.s.. }
$$

(iii) Thirdly, $G$ is, by the way it was constructed, right-continuous a.s.

(iv) Now consider an arbitrary $\mathscr{G}$-measurable random variable $H \geq 0$ a.s.. We wish to see that

$$
G(H(\omega), \omega)=\underset{\xi \in \Xi^{d}(H)}{\operatorname{ess} \sup } \mathbb{E}_{\mathbb{P}}[V(H(\cdot)+\langle\xi(\cdot), Y(\cdot)\rangle, \cdot) \mid \mathscr{G}] \text { a.s. }
$$

This follows immediately when $H$ is a $\mathscr{G}$-measurable countable step-function.

Next, suppose $H$ is any bounded, $\mathscr{G}$-measurable, non-negative (a.s.) random variable, so there exists some $M>0$ such that $H \leq M$ a.s.. It is a well-known fact that we can take a non-increasing sequence $\left\{H_{n} ; n \in \mathbb{N}\right\}$ of $\mathscr{G}_{\text {-measurable step-functions }}$ converging to $H$ a.s., and such that, for every $n \in \mathbb{N}, H_{n} \leq M$ a.s.. Then, fixing an arbitrary $\xi \in \Xi^{d}(H)$, we have for every $n \in \mathbb{N}$ that $H_{n}+\langle\xi, Y\rangle \geq H+\langle\xi, Y\rangle \geq 0$ a.s., therefore

$$
\begin{aligned}
G\left(H_{n}(\cdot), \cdot\right) & =\operatorname{esssup}_{\zeta \in \Xi^{d}\left(H_{n}\right)} \mathbb{E}_{\mathbb{P}}\left[V\left(H_{n}(\cdot)+\langle\zeta(\cdot), Y(\cdot)\rangle, \cdot\right) \mid \mathscr{G}\right] \\
& \geq \mathbb{E}_{\mathbb{P}}\left[V\left(H_{n}(\cdot)+\langle\xi(\cdot), Y(\cdot)\rangle, \cdot\right) \mid \mathscr{G}\right] \text { a.s. }
\end{aligned}
$$

(recall that the equality is true for step-functions), which in turn yields

$$
\liminf _{n \rightarrow+\infty} G\left(H_{n}(\cdot), \cdot\right) \geq \liminf _{n \rightarrow+\infty} \mathbb{E}_{\mathbb{P}}\left[V\left(H_{n}(\cdot)+\langle\xi(\cdot), Y(\cdot)\rangle, \cdot\right) \mid \mathscr{G}\right] \text { a.s. }
$$

But, on the one hand we get by the almost sure path right-continuity of $G$ that $\lim _{n \rightarrow+\infty} G\left(H_{n}(\cdot), \cdot\right)=G(H(\cdot), \cdot)$ a.s.. On the other hand, we can apply the Fatou lemma (see Assumption 4.5) to conclude

$$
\begin{aligned}
\liminf _{n \rightarrow+\infty} \mathbb{E}_{\mathbb{P}}\left[V\left(H_{n}(\cdot)+\langle\xi(\cdot), Y(\cdot)\rangle, \cdot\right) \mid \mathscr{G}\right] & \\
& \geq \mathbb{E}_{\mathbb{P}}[V(H(\cdot)+\langle\xi(\cdot), Y(\cdot)\rangle, \cdot) \mid \mathscr{G}] \text { a.s. },
\end{aligned}
$$

hence $\operatorname{ess} \sup _{\xi \in \Xi^{d}(H)} \mathbb{E}_{\mathbb{P}}[V(H(\cdot)+\langle\xi(\cdot), Y(\cdot)\rangle, \cdot) \mid \mathscr{G}] \leq G(H(\cdot), \cdot)$ a.s. (by the arbitrariness of $\left.\xi \in \Xi^{d}(H)\right)$. Now, to prove the reverse inequality, we can construct (as in part (ii) of this proof) a sequence $\left\{\zeta_{n} ; n \in \mathbb{N}\right\}$ such that, for every $n \in \mathbb{N}$, we have $\zeta_{n} \in \Xi^{d}\left(H_{n}\right), \zeta_{n}(\omega) \in D(\omega)$ for $\mathbb{P}$-a.e. $\omega \in \Omega$, and

$$
\begin{aligned}
\underset{\xi \in \Xi^{d}\left(H_{n}\right)}{\operatorname{ess} \sup _{\mathbb{P}}} \mathbb{E}_{\mathbb{P}}\left[V\left(H_{n}(\cdot)+\langle\xi(\cdot), Y(\cdot)\rangle, \cdot\right) \mid \mathscr{G}\right]-\frac{1}{n} & \\
& \leq \mathbb{E}_{\mathbb{P}}\left[V\left(H_{n}(\cdot)+\left\langle\zeta_{n}(\cdot), Y(\cdot)\right\rangle, \cdot\right) \mid \mathscr{G}\right]
\end{aligned}
$$


We remark further that each $\zeta_{n}$ belongs to $\Xi^{d}(M)$ (because $M+\left\langle\zeta_{n}, Y\right\rangle \geq H_{n}+$ $\left\langle\zeta_{n}, Y\right\rangle \geq 0$ a.s.), so by Lemma 4.7 there exists a random variable $K_{M}$ such that $\left\|\zeta_{n}\right\| \leq \bar{K}_{M}$ a.s.. Therefore we can select a random subsequence $\left\{\zeta_{n_{k}} ; k \in \mathbb{N}\right\}$ with $\lim _{k \rightarrow+\infty} \zeta_{n_{k}}=\zeta$ a.s., for some $\mathscr{G}$-measurable $\zeta$. Clearly,

$$
H+\langle\zeta, Y\rangle=\lim _{k \rightarrow+\infty}\left(H_{n_{k}}+\left\langle\zeta_{n_{k}}, Y\right\rangle\right) \text { a.s. }
$$

and for every $k \in \mathbb{N}$,

$$
H_{n_{k}}+\left\langle\zeta_{n_{k}}, Y\right\rangle=\sum_{i=k}^{+\infty}\left(H_{i}+\left\langle\zeta_{i}, Y\right\rangle\right) \mathbb{1}_{\left\{\omega \in \Omega: n_{k}(\omega)=i\right\}} \geq 0 \text { a.s., }
$$

hence $\zeta \in \Xi^{d}(H)$. Consequently,

$$
\underset{\xi \in \Xi^{d}(H)}{\operatorname{ess} \sup _{\mathbb{P}}} \mathbb{E}_{\mathbb{P}}[V(H(\cdot)+\langle\xi(\cdot), Y(\cdot)\rangle, \cdot) \mid \mathscr{G}] \geq \mathbb{E}_{\mathbb{P}}[V(H(\cdot)+\langle\zeta(\cdot), Y(\cdot)\rangle, \cdot) \mid \mathscr{G}] \text { a.s. }
$$

by definition of essential supremum. Besides, we have by Lemma 4.8 that, for every $k \in \mathbb{N}$,

$$
V^{+}\left(H_{n_{k}(\cdot)}(\cdot)+\left\langle\zeta_{n_{k}(\cdot)}(\cdot), Y(\cdot)\right\rangle, \cdot\right) \leq V^{+}\left(M+\left\langle\zeta_{n_{k}(\cdot)}(\cdot), Y(\cdot)\right\rangle, \cdot\right) \leq L_{M} \text { a.s. }
$$

(note that $\zeta_{n_{k}} \in \Xi^{d}(M)$ ), so Fatou's lemma yields (cf. Assumption 4.4)

$$
\begin{aligned}
\mathbb{E}_{\mathbb{P}}[V(H(\cdot)+\langle\zeta(\cdot), & Y(\cdot)\rangle, \cdot) \mid \mathscr{G}] \\
& \geq \limsup _{k \rightarrow+\infty} \mathbb{E}_{\mathbb{P}}\left[V\left(H_{n_{k}(\cdot)}(\cdot)+\left\langle\zeta_{n_{k}(\cdot)}(\cdot), Y(\cdot)\right\rangle, \cdot\right) \mid \mathscr{G}\right] \\
& \geq \limsup _{k \rightarrow+\infty} G\left(H_{n_{k}(\cdot)}(\cdot), \cdot\right)=G(H(\cdot), \cdot) \text { a.s.. }
\end{aligned}
$$

Combining the inequalities above, we establish (4.7) for any bounded $H$ as well.

Finally, we extend the above result to an arbitrary $\mathscr{G}$-measurable $H \geq 0$ a.s.. Since

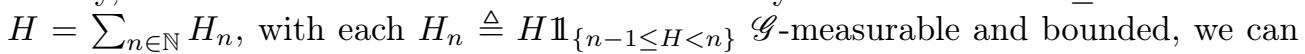
obtain the desired equality from the bounded case.

(v) Lastly, we claim as well that almost all sample paths of $G$ are left-continuous. To see this, let us begin with the remark that, as shown above, for every $x \geq 0$, the function $G(x, \cdot): \Omega \rightarrow \mathbb{R}$, being a version of the essential supremum of $\mathscr{G}$-measurable random variables, is itself measurable with respect to $\mathscr{G}$. In addition, almost every sample path of $G$ is right-continuous. Therefore, by p. 70 of Castaing and Valadier [8], $G:[0,+\infty) \times \Omega \rightarrow \mathbb{R}$ is measurable with respect to the product $\sigma$-algebra $\mathscr{B}([0,+\infty)) \otimes \mathscr{G}$.

Next, defining for every $\omega \in \Omega$,

$$
\bar{G}(x, \omega) \triangleq \begin{cases}\sup _{\substack{q \in \mathbb{Q} \\ q<x}} G(q, \omega), & \text { if } x>0, \\ G(0, \omega), & \text { otherwise }\end{cases}
$$

it is obvious that $\bar{G}$ is $\mathscr{B}([0,+\infty)) \otimes \mathscr{G}$-measurable too. Besides, it is trivial to check that, for every $\omega \in \Omega$, the function $\bar{G}(\cdot, \omega)$ is non-decreasing on $(0,+\infty)$. We remark further that, by construction, all paths of $G$ are left-continuous on $(0,+\infty)$.

It follows immediately from the monotonicity of all the sample paths of $G$ that the inequality $G(x, \omega) \geq \bar{G}(x, \omega)$ holds true for every $x \geq 0$ and $\omega \in \Omega$. In particular, this gives that, for $\mathbb{P}$-a.e. $\omega \in \Omega$ and for all $x \geq 0$, it holds that $\bar{G}(x, \omega)<+\infty$. At last, we shall show that $\mathbb{P}\{\omega \in \Omega: \forall x \geq 0, G(x, \omega)=\bar{G}(x, \omega)\}=1$. 
Non-concave utility maximisation on the positive real axis in discrete time

(a) The proof is by contradiction, so let us suppose that the set

$$
\Omega_{1} \triangleq\{\omega \in \Omega: \exists x>0 \text { s.t. } G(x, \omega)>\bar{G}(x, \omega)\}
$$

has strictly positive measure, i.e., $\mathbb{P}\left(\Omega_{1}\right)>0$. Note that, because $(\Omega, \mathscr{G}, \mathbb{P})$ is a complete measure space, we can apply the measurable projection theorem (see e.g. Theorem III.23 in Castaing and Valadier [8]) to deduce that ${ }^{2}$ $\Omega_{1}=\operatorname{Proj}_{\Omega}\left((G-\bar{G})^{-1}((0,+\infty))\right)$ belongs to $\mathscr{G}$.

Thus, the multi-function $\mathscr{E}: \Omega \rightrightarrows[0,+\infty)$ given by

$$
\mathscr{E}(\omega) \triangleq \begin{cases}\{x>0: G(x, \omega)>\bar{G}(x, \omega)\} & \text { if } \omega \in \Omega_{1} \\ 1, & \text { otherwise }\end{cases}
$$

not only has $\operatorname{dom} \mathscr{E}=\Omega$, but also its graph

$$
\operatorname{gph} \mathscr{E}=\left(\Omega_{1}^{c} \times\{1\}\right) \cup\left(\left[\Omega_{1} \times(0,+\infty)\right] \cap\left[(G-\bar{G})^{-1}((0,+\infty))\right]\right)
$$

is a $\mathscr{G}$-random set. Consequently, we can apply the von Neumann-Aumann theorem to produce a $\mathscr{G}$-measurable selector $H: \Omega \rightarrow[0,+\infty)$ of $\mathscr{E}$. In particular, this implies that

$$
\mathbb{P}\{\omega \in \Omega: G(H(\omega), \omega)>\bar{G}(H(\omega), \omega)\} \geq \mathbb{P}\left(\Omega_{1}\right)>0 .
$$

Also, note that $H>0$. Furthermore, we may and shall assume, without loss of generality, that there exists some $\varepsilon \in(0,1]$ such that $H>\varepsilon$.

(b) On the other hand, we shall see that $G(H(\omega), \omega) \leq \bar{G}(H(\omega), \omega)$ holds for $\mathbb{P}$-a.e. $\omega \in \Omega$, thus contradicting (A.5).

Firstly, fix an arbitrary $n \in \mathbb{N}$. As in part (ii) of this proof, it is possible to construct some $\zeta_{n} \in \Xi^{d}(H)$ such that, for $\mathbb{P}$-a.e. $\omega \in \Omega$,

$$
\mathbb{E}_{\mathbb{P}}\left[V\left(H(\cdot)+\left\langle\zeta_{n}(\cdot), Y(\cdot)\right\rangle, \cdot\right) \mid \mathscr{G}\right](\omega) \geq G(H(\omega), \omega)-\frac{1}{n} .
$$

Next, setting for every $m \in \mathbb{N}$ (recall that $H>\varepsilon$ ),

$$
f_{n}^{m}(\omega) \triangleq V\left(H(\omega)-\frac{\varepsilon}{m}+\frac{H(\omega)-\varepsilon / m}{H(\omega)}\left\langle\zeta_{n}(\omega), Y(\omega)\right\rangle, \omega\right), \quad \omega \in \Omega,
$$

it is trivial by continuity (see Assumption 4.3) that $\left\{f_{n}^{m} ; m \in \mathbb{N}\right\}$ converges a.s. to $V\left(H(\cdot)+\left\langle\zeta_{n}(\cdot), Y(\cdot)\right\rangle, \cdot\right)$, as $m \rightarrow+\infty$. Thus, Fatou's lemma gives

$$
\liminf _{m \rightarrow+\infty} \mathbb{E}_{\mathbb{P}}\left[\left[f_{n}^{m}\right]^{+} \mid \mathscr{G}\right] \geq \mathbb{E}_{\mathbb{P}}\left[V^{+}\left(H(\cdot)+\left\langle\zeta_{n}(\cdot), Y(\cdot)\right\rangle, \cdot\right) \mid \mathscr{G}\right] \text { a.s.. }
$$

Secondly, we note that, for each $m \in \mathbb{N}$, the random vector $\zeta_{n}(H-\varepsilon / m) / H$ belongs to $\Xi^{d}(H-\varepsilon / m)$, because

$$
H-\frac{\varepsilon}{m}+\left\langle\frac{H-\varepsilon / m}{H} \zeta_{n}, Y\right\rangle=\frac{H-\varepsilon / m}{H}\left(H+\left\langle\zeta_{n}, Y\right\rangle\right) \geq 0 \text { a.s. }
$$

(recall that $H>\varepsilon$ and $\zeta_{n} \in \Xi^{d}(H)$ ).

\footnotetext{
${ }^{2}$ Given a set $E \subseteq X \times Y$, we recall that the projection of $E$ on $X$ is$$
\operatorname{Proj}_{X}(E) \triangleq\{x \in X: \exists y \in Y \text { such that }(x, y) \in E\},
$$ 
Therefore, given Assumption 4.5 and the fact that, for every $m \in \mathbb{N}$, the inequality $\left[f_{n}^{m}\right]^{-} \leq V^{-}(0, \cdot)$ is true a.s., we can apply the Fatou lemma to obtain

$$
\limsup _{m \rightarrow+\infty} \mathbb{E}_{\mathbb{P}}\left[\left[f_{n}^{m}\right]^{-} \mid \mathscr{G}\right] \leq \mathbb{E}_{\mathbb{P}}\left[V^{-}\left(H(\cdot)+\left\langle\zeta_{n}(\cdot), Y(\cdot)\right\rangle, \cdot\right) \mid \mathscr{G}\right] \text { a.s.. }
$$

Combining both inequalities yields

$$
\liminf _{m \rightarrow+\infty} \mathbb{E}_{\mathbb{P}}\left[f_{n}^{m} \mid \mathscr{G}\right] \geq \mathbb{E}_{\mathbb{P}}\left[V\left(H(\cdot)+\left\langle\zeta_{n}(\cdot), Y(\cdot)\right\rangle, \cdot\right) \mid \mathscr{G}\right] \text { a.s.. }
$$

Besides,

$$
\operatorname{ess~sup}_{\xi \in \Xi^{d}(H-\varepsilon / m)} \mathbb{E}_{\mathbb{P}}\left[V\left(H(\cdot)-\frac{\varepsilon}{m}+\langle\xi(\cdot), Y(\cdot)\rangle, \cdot\right) \mid \mathscr{G}\right] \geq \mathbb{E}_{\mathbb{P}}\left[f_{n}^{m} \mid \mathscr{G}\right] \text { a.s. }
$$

for every $m \in \mathbb{N}$, and so

$$
\begin{aligned}
& \liminf _{m \rightarrow+\infty} \operatorname{essipu}_{\xi \in \Xi^{d}(H-\varepsilon / m)} \mathbb{E}_{\mathbb{P}}\left[V\left(H(\cdot)-\frac{\varepsilon}{m}+\langle\xi(\cdot), Y(\cdot)\rangle, \cdot\right) \mid \mathscr{G}\right] \\
& \geq \liminf _{m \rightarrow+\infty} \mathbb{E}_{\mathbb{P}}\left[f_{n}^{m} \mid \mathscr{G}\right] \text { a.s.. }
\end{aligned}
$$

On the other hand, let $m \in \mathbb{N}$ be arbitrary, but fixed. Then we know by the preceding step that

$$
\begin{aligned}
\operatorname{esssup}_{\xi \in \Xi d(H-\varepsilon / m)} \mathbb{E}_{\mathbb{P}}\left[V\left(H(\cdot)-\frac{\varepsilon}{m}+\langle\xi(\cdot), Y(\cdot)\rangle, \cdot\right) \mid \mathscr{G}\right](\omega) & \\
& =G\left(H(\omega)-\frac{\varepsilon}{m}, \omega\right)
\end{aligned}
$$

for every $\omega$ outside a $\mathbb{P}$-null set. Next, choosing $q_{m} \in \mathbb{Q}, q>0$ such that $H(\omega)-\varepsilon / m \leq q_{m}<H(\omega)$, it follows immediately from the definition of $\bar{G}$ (recall that $H>\varepsilon>0$ ) and from the monotonicity of $G$ (see the first part of this proof) that

$$
\begin{aligned}
\bar{G}(H(\omega), \omega)=\sup _{\substack{q \in \mathbb{Q} \\
q<H(\omega)}} G(q, \omega) & \geq G\left(q_{m}, \omega\right) \\
& \geq G(H(\omega)-\varepsilon / m, \omega) \geq \inf _{k \geq m} G(H(\omega)-\varepsilon / k, \omega),
\end{aligned}
$$

consequently,

$$
\bar{G}(H(\cdot), \cdot) \geq \sup _{m \in \mathbb{N}} \inf _{k \geq m} G(H(\cdot)-\varepsilon / k, \cdot)=\liminf _{m \rightarrow+\infty} G(H(\omega)-\varepsilon / m, \omega) \text { a.s.. }
$$

So, putting together all the inequalities above finally yields that, for every $n \in \mathbb{N}$, $\bar{G}(H(\cdot), \cdot) \geq G(H(\cdot), \cdot)-1 / n$ a.s., hence

$$
\bar{G}(H(\cdot), \cdot) \geq \limsup _{n \rightarrow+\infty}\left(G(H(\cdot), \cdot)-\frac{1}{n}\right)=G(H(\cdot), \cdot) \text { a.s. }
$$

as claimed.

Proof of Proposition 4.10. Let $\xi_{n} \in \Xi^{d}(H)$ be a sequence attaining the essential supremum. We may assume $\xi_{n} \in D$ a.s. and hence $\left|\xi_{n}\right| \leq H / \beta$ a.s. for all $n$, by Lemma 4.7 . Lemma 2 of Kabanov and Stricker [12] implies the existence of a $\mathscr{G}$-measurable random subsequence $n_{k}$ such that $\xi_{n_{k}} \rightarrow \xi$ a.s., $k \rightarrow \infty$. Lemma 4.8 allows the use of the (conditional) Fatou lemma hence we get that $\tilde{\xi}(H):=\xi$ is as claimed. 


\section{References}

[1] Berkelaar, A. B.; Kouwenberg, R.; Post, T. (2004). Optimal portfolio choice under loss aversion. Rev. Econom. Statist. 86, no. 4, pp. 973-987.

[2] Bernard, C.; Ghossoub, M. (2010). Static portfolio choice under Cumulative Prospect Theory. Math. Financ. Econ. 2, no. 4, pp. 277-306.

[3] Campi, L., Del Vigna, M. (2011). Weak insider trading and behavioural finance. SIAM J. Financial Math., 3, pp. 242-279.

[4] Carassus,L. and Pham, H. (2009). Portfolio optimization for nonconvex criteria functions. RIMS Kôkyuroku series, ed. Shigeyoshi Ogawa, 1620, pp. 81-111, 2009.

[5] Carassus, L.; Rásonyi, M. (2014). Maximization of non-concave utility functions in discrete-time financial market models. Submitted. Available online at http://arxiv.org/abs/1302.0134v2

[6] Carassus, L.; Rásonyi, M. (2013). On optimal investment for a behavioral investor in multiperiod incomplete market models. Math. Finance, published online. doi: $10.1111 /$ mafi. 12018

[7] Carlier, G; Dana, R.-A. (2011). Optimal demand for contingent claims when agents have law invariant utilities. Math. Finance 21, no. 2, pp. 169-201.

[8] Castaing, C.; Valadier, M. (1977). Convex analysis and measurable multifunctions. Lecture Notes in Mathematics 580. Springer-Verlag.

[9] He, X. D.; Zhou, X. Y. (2011). Portfolio choice under cumulative prospect theory: an analytical treatment. Management Sci. 57, no. 2, pp. 315-331.

[10] Jacod, J.; Shiryaev, A. N. (1998). Local martingales and the fundamental asset pricing theorems in the discrete-time case. Finance Stochast. 2, no. 3, pp. 259-273.

[11] Jin, H.; Zhou, X. Y. (2008). Behavioral portfolio selection in continuous time. Math. Finance 18, no. 3, pp. 385-426.

[12] Kabanov, Y.; Stricker, C. (2001). A teacher's note on no-arbitrage criteria. In: Azéma, J.; Émery, M.; Ledoux, M.; Yor, M. (Eds.), Séminaire de Probabilités XXXV, Lecture Notes in Mathematics 1755, pp. 149-152. Springer-Verlag.

[13] Kahneman, D.; Tversky, A. (1979). Prospect theory: An analysis of decision under risk. Econometrica 47, no. 2, pp. 263-292.

[14] Kramkov, D.; Schachermayer, W. (1999). The asymptotic elasticity of utility functions and optimal investment in incomplete markets. Ann. Appl. Probab. 9, no. 3, pp. 904950 .

[15] Rásonyi, M.; Rodrigues, A. M. (2013). Optimal portfolio choice for a behavioural investor in continuous-time markets. Ann. Finance 9, no. 2, pp. 291-318.

[16] Rásonyi, M.; Rodrigues, A. M. (2014). Continuous-time portfolio optimisation for a behavioural investor with bounded utility on gains. Electron. Commun. Probab. 19, no. 38 , pp. $1-13$.

[17] Rásonyi, M.; Rodríguez-Villarreal, J. G. (2013). Optimal investment under behavioural criteria - a dual approach. To appear in Banach Center Publications. Available online at http://arxiv.org/abs/1405.3812 
[18] Rásonyi, M.; Stettner, Ł. (2005). On utility maximization in discrete-time financial market models. Ann. Appl. Probab. 15, no. 2, pp. 1367-1395.

[19] Rásonyi, M.; Stettner, Ł. (2006). On the existence of optimal portfolios for the utility maximization problem in discrete time financial market models. In: Kabanov, Y.; Liptser, R.; Stoyanov, J. (Eds.), From Stochastic Calculus to Mathematical Finance, pp. 589-608. Springer.

[20] Reichlin, C. (2013). Utility maximization with a given pricing measure when the utility is not necessarily concave. Math. Financ. Econ. 7, no. 4, pp. 531-556.

[21] Reichlin, C. (2012). Non-concave utility maximization: optimal investment, stability and applications (PhD thesis). ETH Zürich, Switzerland.

[22] Tversky, A.; Kahneman, D. (1992). Advances in prospect theory: cumulative representation of uncertainty. Journal of Risk and Uncertainty 5, no. 4, pp. 297-323.

[23] von Neumann, J.; Morgenstern, O. (1944). Theory of games and economic behavior. Princeton University Press. 